\title{
Package Capacity
}

National Cancer Institute

\section{Source}

National Cancer Institute. Package Capacity. NCI Thesaurus. Code C93887.

The maximum number of product units within a package. 